\title{
AN INTEGER PROGRAMMING MODEL FOR ANALYSING IMPACTS OF DIFFERENT TRAIN TYPES ON RAILWAY LINE CAPACITY
}

\author{
Masoud Yaghini, Nariman Nikoo, Hamid Reza Ahadi \\ Dept of Rail Transportation Engineering, School of Railway Engineering, \\ Iran University of Science and Technology, Tehran, Iran
}

Submitted 9 May 2012; accepted 7 September 2012

\begin{abstract}
The evaluation of railway line capacity is an important problem, which effects majority of problems in rail transportation planning. The railway capacity is dependent on infrastructure, traffic, and operating parameters. A key factor affecting railway line capacity is the impact of different train types. As the combination of different train types increases, more interference is generated. In this paper, for evaluation of train type interactions on railway line capacity, an integer-programming model for both line and line section is presented. The problem is formulated as a multicommodity network design model on a space-discrete time network. The railway capacity is calculated using data typically available to planners. The inputs of the model are the characteristic of each train type and railway line attributes. The model determines railway capacity based on train type mixes. In addition, this model considers impact of train types on capacity and waiting time. In order to show the features of the model, a case study is implemented in Iran Railways. The capacity tends to increase non-linearly with small incremental changes in parameters. The mixture of train types reduces the railway line capacity. The proposed model can help railway managers for long-term planning.
\end{abstract}

Keywords: railway transportation, railway capacity, railway line, line section, siding track, discrete-time multicommodity network flow model, integer programming.

Reference to this paper should be made as follows: Yaghini, M.; Nikoo, N.; Ahadi, H. R. 2014. An integer programming model for analysing impacts of different train types on railway line capacity, Transport 29(1): 28-35.

http://dx.doi.org/10.3846/16484142.2014.894938

\section{Introduction}

The main goal of railway transport is to attract a maximum number of customers and load on planned or existing lines with a minimum of investment cost, personnel, equipment, energy consumption, operating and maintenance costs (Hansen 2010).

Railway line capacity depends on railway infrastructure and timetable and, thereby, implicitly on the rolling stock used; and it can be measured with given actual infrastructure and timetable (Landex 2009). Capacity may be different for each proportional mix of trains (train types) and the order in which they run on the line (Burdett, Kozan 2006).

To invest the capital efficiently, understanding the different operational characteristics of the traffic is required (Dingler et al. 2009). Railway lines have different train types including passenger and freight trains. The ideal case is when all trains are the same or have the same velocity. More interference is generated by increasing the mixture of different trains. Thus, trains require overtaking and crossing, and the mixture reduces the traffic flow. Each of these train types can have significantly different characteristics and even trains of the same class may have varying weights and lengths. This heterogeneity has a substantial effect on rail line capacity (Dingler et al. 2009). In addition, the methods of line capacity calculation usually require a built timetable, which is not usually available in the planning stage. Therefore, it is necessary to develop procedures to evaluate railway line capacity by considering heterogeneity without existing timetable.

We perform analysis using mathematical modelling to determine the impacts of different train types on railway line capacity.

The main contribution of this research is to present an optimization model for evaluation of the interaction of different train types on railway line and line section capacity by using data typically available to planners. It

Corresponding author: Masoud Yaghini

E-mail: yaghini@iust.ac.ir 
means that no detailed timetable is needed. We make the following contributions to the field:

- an integer programming model for calculation and evaluation of railway line and line section capacity;

- an optimization model to evaluate the interaction of different train types on railway lines and line sections considering train type priority;

- formulating the problem as a multicommodity network flow model on the space-time network;

- presenting a case study in Iran Railways as a real life problem.

In this paper, the aim of the capacity problem analysis is to maximize the number of train paths over a rail line in the reference time. The inputs of the model are infrastructure (line) and train type attributes. The outputs of the proposed model are line capacity for each line section. For capacity calculation, first, the possible paths that consist of some arcs are built. Subsequently, the railway capacity model is formulated using multicommodity flow model. This model considers infrastructure, traffic and operating parameters simultaneously. The model takes care of train overtaking and crossing caused by train with different speed mix.

The paper is organized as follows. A review of the related literature is presented in Section 1. The proposed railway capacity model for evaluation of the interaction of different train types on railway line capacity is explained in Section 2. In Section 3, an example is described and the capacity model is solved. Iran Railways capacity is calculated and evaluated as a case study and the results are reported in Section 4. Last section provides the conclusions.

\section{Literature Review}

Several methods have been developed to calculate and evaluate railway line capacity. These methods can be classified in four categories: analytical, parametric, optimization and simulation. Analytical methods such as International Union of Railways (UIC 2004), are designed to model the railway environment by using mathematical formulae or algebraic expressions. They usually obtain theoretical capacities and determine practical capacities either as a percentage of the theoretical capacity or by including regularity margins. Parametric methods model capacity by parametric relationships between infrastructure, traffic and operating parameters. Simulation methods, such as RailSys, simulate the running operation and evaluate the capacity (Khadem-Sameni et al. 2010). Optimization methods are based on obtaining optimal saturated timetables. These optimal timetables are usually obtained by using mathematical programming techniques (Abril et al. 2008).

Analytical methods are quick but they provide limited information for capacity evaluation. It is conducted by calculation of minimum headways from the infrastructure and timetable characteristics to determine and describe the capacity of a line or other parts of a railway line. Simulation models are more realistic but data in- tensive and computationally difficult. Parametric models, which come in between analytical and simulation models, are very sensitive to parameter inputs and train mix variations (Abril et al. 2008; Pachl, White 2004; Khadem-Sameni et al. 2010). Optimization methods are designed to provide more strategic perspective for solving the railway capacity problem and consider various parameters that affect railway line capacity. Combinatorial optimization models are used more and more for strategic line planning in large complex networks, timetable design, rolling stock and crew scheduling. The models aim to solve the timetable problem for a certain objective function under predefined constraints to optimality and, thus, generate an optimal design for the individual departure and arrival times in a network (Hansen 2010). The literature review is divided in two subsections that include models for determination of railway capacity and models that evaluate the impact of train types on rail line capacity. These subsections are discussed as follows.

\subsection{Models for Determination of Railway Capacity}

In this section, the optimization methods that focus on capacity evaluation are reviewed. Based on the actual infrastructure and timetable, UIC 406 capacity leaflet gives a method to measure the capacity consumption of line sections. This method defines a methodology to measure the capacity consumption based on compressing timetable graphs (UIC 2004).

Merel et al. (2009) consider the problem of measuring the capacity of the Pierrefitte-Gonesse junction, an important crossing point for various types of traffic in France. In their paper, capacity is evaluated by solving an optimization problem called saturation problem. It entails routing maximum number of trains through the infrastructure, possibly allowing delays but preventing safety-related conflicts and other practical exploitation constraints. The binary decision variables of this model show activated trains on route with a delay relative to their nominal time.

Libardo et al. (2011) describe an improved Station Layout Computing (SLC) model that identifies the maximum capacity of a railway junction while respecting constraints due to the junction layout and the predefined timetable. This method follows the main guidelines defined in UIC 406 capacity leaflet, which aims at calculating the capacity of railway lines. Libardo et al. (2011) find the solution that minimizes the total compressed time. The optimal solution of this optimization step identifies the shortest possible time window for satisfying the timetable. The optimal value of the decision variables is an input of the second optimization step. The second optimization step determines the maximum capacity of the junction in terms of frequencies offered in the time horizon while satisfying the timetable in the shortest possible time window.

Burdett and Kozan (2006) apply the general approaches for determining absolute capacity in railways based on the logic of an existing bottleneck approach. 
The approaches presented in this paper are suitable for railway lines and networks with uni- and/or bi-directional traffic, and do not require any major modifications when dealing with one scenario or another. For railway lines, the absolute capacity is the number of trains that could traverse the critical section. The absolute capacity of a network is similar to the line one. It is the total number of trains that traverse all corridors. The other variables signifying the numbers travelling across individual sections are therefore redundant. The main approach developed in Burdett and Kozan (2006) is a generic optimization approach that is determined by solving an optimization model. The objective in particular is the total throughput between all pairs of Input-Output (IO) locations.

\subsection{Models for Evaluation of Impact of Train Types on Rail Line Capacity}

Key elements with direct influence on the value of the capacity are geometrical configuration of the tracks, line and stations and their layout, features of signalling systems, movement rules and corresponding minimum distance between trains, operation and maintenance planning and quality measures (Kontaxi, Ricci 2011). Abril et al. (2008) demonstrate an increase in singletrack line capacity when faster trains are introduced into the schedule. Capacity is measured as trips completed. Dingler et al. (2009) provide a better understanding of the impacts of various aspects of train type heterogeneity to enable more effective planning and efficient rail operations by using simulation software. The results also suggest certain operating strategies that may reduce the delays caused by train type heterogeneity. Harrod (2009) presents the utility maximizing problem. This problem is a multicommodity flow problem consisting of a linear network model with side constraints enforcing the operational interaction of separate trains. The objective value of the formulated problem represents an aggregate utility of the dispatch plan upon the given network. Capacity is measured as the number of valid train paths over a fixed time horizon within an optimal master schedule. The utility in this study is defined as a linear function of the number of completed train paths, and additional network time consumed beyond the plan time horizon. Harrod (2009) evaluates the fiftyfour combinations of track network and speed differential. Landex et al. (2006) analyse the Dutch rail system, but focus on the importance of line segment length in determining capacity with heterogeneous traffic. Lai and Barkan (2011) present a framework with alternatives generator, investment selection model and impact analysis module tools for strategic railway capacity planning. Alternatives generator enumerates the possible expansion options along with their cost and capacity effects. Investment selection model determines the portions of the network that need to be upgraded with certain capacity improvement alternatives. Moreover, impact analysis module evaluates the trade-off between capital investment and delay cost.

\section{Proposed Optimization Model}

Given the operational conditions, the railway line capacity determines the maximum number of trains that would be able to operate on a given railway infrastructure during a specific time interval (Abril et al. 2008).

In the proposed model, three steps for calculation of railway line capacity are defined. In the first step, input data from infrastructure and train type attributes readied. In step two, basic input data and the space-time network is created. The output of this step is travel and waiting arcs. Then, in the last step, an integer-programming model based on the space-discrete time network is solved.

\subsection{Model Inputs}

There are two input categories, namely, railway line and train type attributes.

\subsubsection{Railway Line Attributes}

Railway line attributes explain the routes and nodes of railway line. In order to define the railway line, four attributes, namely, origin node, destination node, lengths of the line section, and number of tracks in each line section are needed. A route is a consecutive set of lines and nodes between a defined source and target. Line is a link between two large nodes and usually the sum of more than one line section. A line section is the part of a line in which the traffic mix and/or the number of trains or the infrastructure and signalling conditions do not change fundamentally. It consists of one or more coherent section, which is limited by two neighbours stations or nodes. A set of station nodes is defined as $S N=\{1$, $2, \ldots\}$ and a set of line sections is defined as $L S=\{1-2$, $2-3, \ldots\}$. NT indicates the number of tracks in each line sections. $i, j$, and $p$ represent node indices.

\subsubsection{Train Type Attributes}

All trains that have the same average velocity and direction belong to the same train type. Train type attributes are train origin, train destination, and the average velocity of the train. Train type index is defined as $r$ that belongs to $R$. The set of train types is $R=\{1,2, \ldots\}$. $R^{\text {north }}$ shows north trains and $R^{\text {south }}$ shows south trains so that $R^{\text {north }} \cup R^{\text {south }}=R$. A siding track is an auxiliary track for meeting or passing trains. $n s_{i}$ is the number of siding tracks that can receive trains simultaneously. $t m p_{r}$ stands for percentage of train type $r$ in railway traffic. Let $d_{r}$ denote the destination of train type $r, o_{r}$ origin of train type $r$, and $p d_{r}$ previous station of train type $r$. The priorities of trains play a critical role. Train priorities decrease capacity because priority trains are given preferential treatment over lower priority trains, which in turn results in increased delays. This allows the priority traffic to move as if it were the only traffic in the line. $p^{r}$ stands for priority of train type $r$ in railway traffic.

\subsection{The Space-Time Network}

In this paper, a space-time network developed for representation of railway line. In this step and based on input data, train paths and decision variables are built. 
This space-time network representation reduces extra number of decision variables. As shown in Fig. 1, the space-time network is represented as $G=(N, A)$, where $N$ denotes the time-node set and $A$ denotes the arc set. Each train type is a commodity in the network. Time is separated into discrete time horizons with equal time intervals between consecutive time instants. Nodes are defined for each node and each instant time. The set $A$ of arcs is composed of two subsets: travel arcs and waiting arcs. Since each train in each time unit travels on the line sections or waits in the nodes, two sets of arcs are defined. Travel arcs represent the occupancy of a line section between two stations (nodes) over an interval time units. Waiting arcs represent stopping times at the stations or sidings. In the space-time network, the travel arcs and the waiting arcs are created for each train type and in each discrete time.

Time window is the interval or unit of time that is taken as a reference for computing the desired line capacity. Traditionally, this is fixed either to one hour or to the whole working day (UIC 2004). Let $T$ be chosen discrete times in minutes. The discrete time horizon is categorized with starting value $t=1$. Let the set of time instants be defined as $T=\{1,2, \ldots, t\} . l$ and $m$ represent time indices. The time it takes to traverse a given line section is the running time. Since train length does not have significant effects on capacity analysis, we do not consider it in running time computation. Each arc is shown with $X_{i, j, l, m}^{r}$ that denote the train $r$ dispatches from node $i$ at time $l$ and receives at node $j$ at time $m$. For building travel arcs, they are created for each train type and from origin node to destination node for each time period $t$ using the average velocity of train type and length of line section, which is less than the difference between time windows and running time. For example, $X_{1,2,0,2}^{r}$ shows train $r$ dispatched from node 1 at time 0 and received in node 2 at time 2 . This binary variable belongs to set of travel arcs. If this variable is equal to 1 , it means the line section consists of node 1 and 2 occupied in time 0 to 2 . The proposed algorithm for building travel arcs calculate the receiving time of train $r$ in each line section and time instant with considering the average velocity and length of line sections. This algorithm creates a set of travel arcs.

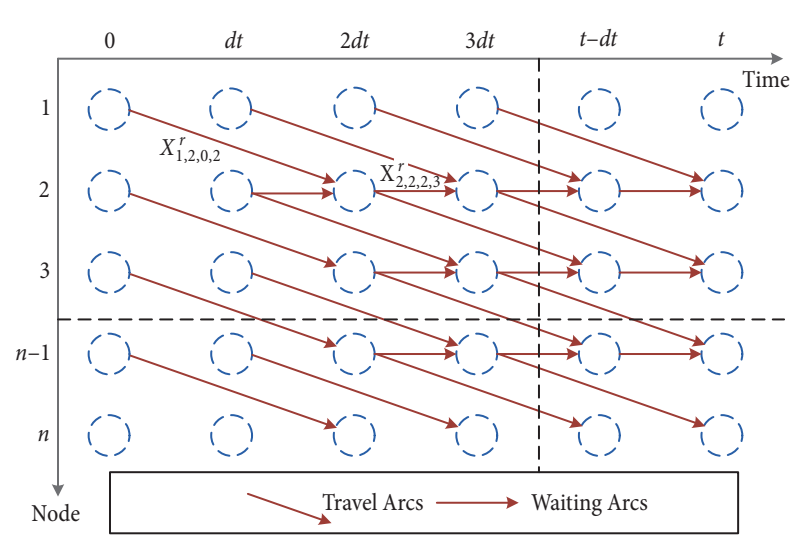

Fig. 1. Space-time network
Waiting arcs are created for each train type, intermediate node, time period $t$ that is less than the difference between time window and running time, and for each siding track. For example, $X_{2,2,2,3}^{r}$ shows train $r$ dispatched from node 2 at time 2 and received in node 2 at time 3 . This binary variable belongs to the set of waiting arcs because dispatching node and receiving node is one node. If this variable is equal to 1 , it means that train $r$ waited in node 2 from time 2 to time 3 .

The consecutive arcs of travel and waiting arcs build a train path. The binary variables of consecutive arcs for each train equal to one indicate a complete journey of train $r$ from its origin to its destination. The outputs of this step are arcs with five attributes, namely, train type, dispatching time, receiving time, dispatching node and receiving node. This arc set is the decision variables of the model.

For capacity evaluation, first, possible train paths from railway line and train type attributes consisting of arcs are built. Train arcs are built in this step. Then, based on feasible arcs, the railway capacity model as a discrete-time multicommodity network flow problem is formulated. The model is coded in the Java programming language and using CPLEX software. In the following subsections, the capacity calculation for railway line capacity and line section capacity are described.

\subsection{Capacity Calculation for Railway Line}

In this step, based on the arcs built in previous step, a model for railway line capacity problem is proposed. The suggested model is formulated as a multi-commodity network flow model problem with binary decision variables. The capacity of a line is the maximum number of trains arriving at their destinations taking into account each priority. To consider the railway line characteristics, the following formulation is used:

$$
\begin{aligned}
& M A X=\sum_{r} \sum_{l} \sum_{m} p^{r} X_{p d_{r}, d_{r}, l, m}^{r} \\
& \sum_{i} \sum_{l} X_{i, j, l, m}^{r}-\sum_{i} \sum_{m} X_{j, i, l, m}^{r}=0, \\
& \forall r \in R, \forall j \in S N \forall l, m \in T ;
\end{aligned}
$$

$\sum_{r \in R^{\text {south }}} \sum_{l \leq k-1} \sum_{m \geq k} X_{i,(i+1), l, m}^{r}+\sum_{r \in R^{\text {north }}} \sum_{l \leq k-1} \sum_{m \geq k} X_{(i+1), i, l, m}^{r} \leq N T$,

$\forall k \in T, \forall i \in L S$;

$\sum_{r} X_{i, i, l, l+1}^{r} \leq n s_{i}$

$\forall l \in T, \forall i \in S N, i \neq d_{r}, i \neq o_{r} ;$

$\sum_{l} \sum_{m} X_{p d_{r}, d_{r}, l, m}^{r} \geq t m p_{r} \sum_{r} \sum_{l} \sum_{m} X_{p d_{r}, d_{r}, l, m}^{r}$,

$\forall r \in R$;

$X_{i, j, l, m}^{r} \in\{0,1\}, \forall l, m \in T, \forall i, j \in S N, \forall r \in R$.

The objective function maximizes the number of train paths over a rail line by considering the priorities. As a rule, the greater the number of priority classes, the less capacity is available. Railway line capacity is determined by the equation (1). On double-track lines, overtaking issue and on single-track lines, the overtaking and 
crossing issues exist. Single-track lines can only operate the trains in one direction at a time, but operation of the trains on double-track lines in two directions at a time is possible. These issues are considered with constraint sets (2) and (3). Node sequence of each train in each time is continuous. Constraint (2) provides a set of flow conservation constraints for every node in discrete time units. Any train therefore entering these nodes should also leave them. Constraint set (3) is the line section constraints considering north and south trains. These constraints guarantee that at any time interval there is no more than NT trains traveling on a track section. Constraint set (4) ensures that at any time interval, the number of waiting trains at a station or a siding does not exceed the number of receiving tracks of the station or the siding. Railway capacity is dependent on traffic mix. For considering each proportional mix of trains in the railway line, constraint set (5) is added to the model. This expression guarantees that the minimum proportional mix of trains for each train type traverses on the railway line. The decision variable set of this model is $X_{i, j, l, m}^{r}$ which is binary. More constraints can be added to the model in different conditions. The operation pattern of double track lines should normally be such that each track is reserved for one direction of travel. For considering this operation pattern, constraint set (3) is removed from model and the following expressions can be added to the model. These constraints ensure that each track is reserved for one direction of travel, southbound or northbound:

$$
\begin{aligned}
& \sum_{r \in R^{\text {south }}} \sum_{l \leq k-1} \sum_{m \geq k} X_{i,(i+1), l, m}^{r} \leq 1, \forall l, m \in T, \forall i \in L S ; \\
& \sum_{r \in R^{\text {north }}} \sum_{l \leq k-1} \sum_{m \geq k} X_{(i+1), i, l, m}^{r} \leq 1, \forall l, m \in T, \forall i \in L S .
\end{aligned}
$$

\subsection{Capacity Calculation for Railway Line Section}

After determination of line capacity in previous section, the number of trains that can traverse in each line section can be obtained. So, one can calculate capacity of each line section and percentage of each train type. The capacity of railway line section $(i-j)$ can be separately determined by the following proposed equation:

$$
\sum_{r} \sum_{l} \sum_{m} X_{i, j, l, m}^{r}+\sum_{r} \sum_{l} \sum_{m} X_{j, i, l, m}^{r} .
$$

If evaluation of the interaction of different train types on the critical line section (line section with maximum running time) is considered separately, the following constraints are used. In this approach, constraint sets 2 and 4 are removed from railway line constraints. The objective function maximizes the number of train paths over a discrete time rail line in each line section. Railway line capacity is determined by equation (10). The objective function maximizes the number of train paths over a rail line section by considering priorities. Railway line section capacity in line section $(i-j)$ is determined by the following equations:

$$
M A X=\sum_{r} \sum_{l} \sum_{m} p^{r}\left(X_{i, j, l, m}^{r}+X_{j, i, l, m}^{r}\right) ;
$$

$$
\begin{aligned}
& \sum_{r \in R^{\text {south }}} \sum_{l \leq k-1} \sum_{m \geq k} X_{i,(i+1), l, m}^{r}+\sum_{r \in R^{\text {north }}} \sum_{l \leq k-1} \sum_{m \geq k} X_{(i+1), i, l, m}^{r} \leq N T, \\
& \forall k \in T, \forall i \in L S ; \\
& \sum_{l} \sum_{m}\left(X_{i, j, l, m}^{r}+X_{j, i, l, m}^{r}\right) \geq t m p_{r} \sum_{r} \sum_{l} \sum_{m}\left(X_{i, j, l, m}^{r}+X_{j, i, l, m}^{r}\right), \\
& \forall r \in R ; \\
& X_{i, j, l, m}^{r} \in\{0,1\}, \forall l, m \in T, \forall i, j \in S N, \forall r \in R .
\end{aligned}
$$

Constraint (11) is a set of line section constraints with separation of north and south trains. These constraints guarantee that at any time interval there is no more than NT train traveling on a line section. Constraint (12) guarantees that the minimum proportional mix of trains for each train type traverses on the line section.

\section{A Case Study}

To demonstrate the application of the proposed model, Tehran-Zanjan route is considered (Fig. 2). This singletrack railway line has 21 stations with a total of 332 kilometre. Table 1 shows the length of each line section from node 1 (Tehran) to 21 (Zanjan). For each station, one siding track is considered that allows one train to wait clearance of main track.

The ideal situation is when all trains are the same or have the same speed. For capacity evaluation, first, we consider the whole route and only one train type travels from north to south. In this case, the average velocities of trains change from 80 to $240 \mathrm{~km} / \mathrm{h}$ while all other parameters were held constant. Time window is discrete in 5 minutes, and there is 1440 minutes in each day. For each average velocity, capacity, the average waiting time for each train and total waiting time are calculated. As shown in Table 2, the number of arcs produced in each scenario with increasing of the average velocity is increased. With increasing of the average velocity, the running time of trains in each line section is decreased and it causes decreasing of headway of two consecutive trains. The results are given in Table 2. This problem is solved using CPLEX software. Total waiting time shows summation of waiting time of total trains in all siding tracks of nodes and stations. The average waiting time shows the average waiting time of each train in the all siding tracks of the route.

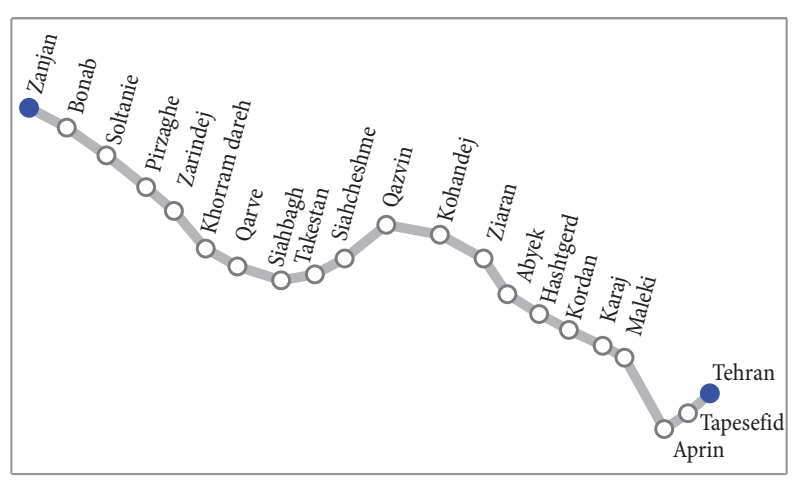

Fig. 2. Tehran-Zanjan route in Iran 
Table 1. Tehran-Zanjan route attributes

\begin{tabular}{ccc}
\hline From node & To node & Length $(\mathrm{km})$ \\
\hline 1 & 2 & 10 \\
\hline 2 & 3 & 10 \\
\hline 3 & 4 & 22 \\
\hline 4 & 5 & 17 \\
\hline 5 & 6 & 18 \\
\hline 6 & 7 & 17 \\
\hline 7 & 8 & 17 \\
\hline 8 & 9 & 16 \\
\hline 9 & 10 & 17 \\
\hline 10 & 11 & 18 \\
\hline 11 & 12 & 18 \\
\hline 12 & 13 & 16 \\
\hline 13 & 14 & 16 \\
\hline 14 & 15 & 16 \\
\hline 15 & 16 & 17 \\
\hline 16 & 17 & 17 \\
\hline 17 & 18 & 16 \\
\hline 18 & 19 & 20 \\
\hline 19 & 20 & 17 \\
\hline 20 & 21 & \\
\hline
\end{tabular}

Railway line capacity of this route versus the average velocity is shown in Fig. 3. For instance, if the average velocity is equal to $160 \mathrm{~km} / \mathrm{h}$, the railway line capacity is 132 trains per day. As shown in Figs 3 and 4, and as expected, increasing the average velocity of trains, capacity is increased, but waiting time increases and then decreases. Lower speed trains decrease the railway line capacity because they need more running time.

Fig. 5 illustrates the train timetable diagram using the proposed method for the trains with an average velocity of $120 \mathrm{~km} / \mathrm{h}$. The horizontal and vertical axes represent time and stations, respectively. In this graph, each path shows a train. In this scenario, the trains have the highest waiting time. There are 86 trains moving from node 1 (Tehran) to node 21 (Zanjan).

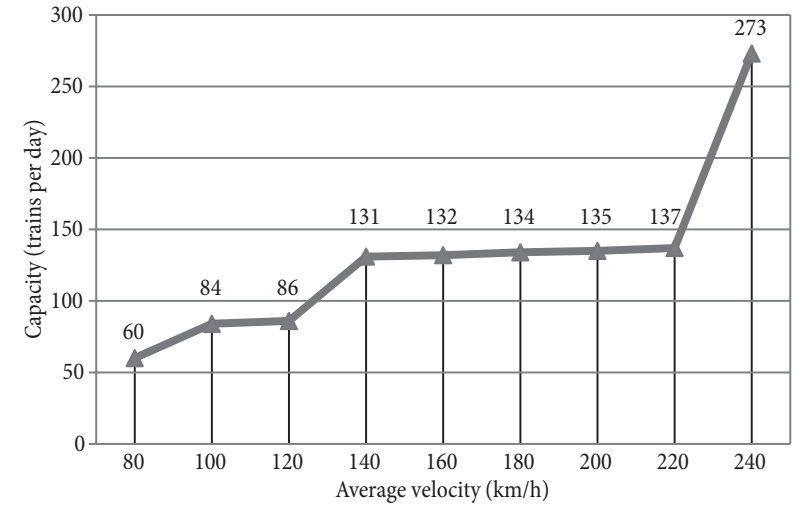

Fig. 3. The capacity-velocity

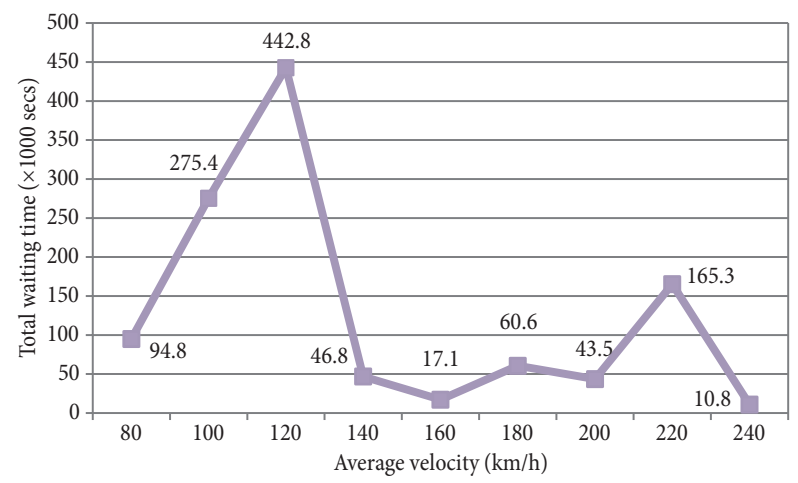

Fig. 4. Total waiting time

Railway capacity is very much dependent on traffic mix. To continue, the critical line section (line section with maximum running time) that is Aprin-Maleki, and four train types are considered with the average velocities of $80,78,100$ and $102 \mathrm{~km} / \mathrm{h}$. In this case, the impact of train type mix on railway capacity is evaluated. Time window is set to for the whole day, that is, 1440 minutes. Time window is discrete in 1 minute. The capacity of the critical line section with proportional mix of train types is shown in Table 3. Line section capacity of TehranZanjan route is equal to 117 trains per day. It consists of 13 trains of type $3(100 \mathrm{~km} / \mathrm{h}$ trains $)$ and 104 trains of

Table 2. Line capacity evaluation

\begin{tabular}{ccccc}
\hline Average velocity $(\mathrm{km} / \mathrm{h})$ & Number of variables & Capacity (trains per day) & $\begin{array}{c}\text { Total waiting } \\
\text { time }(\times 1000 \text { seconds) }\end{array}$ & $\begin{array}{c}\text { Average waiting time for } \\
\text { each train }(\times 1000 \text { seconds })\end{array}$ \\
\hline 80 & 18539 & 60 & 94.8 & 1.58 \\
\hline 100 & 19141 & 84 & 275.4 & 5.28 \\
\hline 120 & 19527 & 86 & 442.8 & 0.15 \\
\hline 140 & 19912 & 131 & 46.8 & 0.13 \\
\hline 160 & 20130 & 132 & 17.1 & 0.45 \\
\hline 180 & 20303 & 134 & 60.6 & 0.32 \\
\hline 200 & 20424 & 135 & 165.3 & 1.21 \\
\hline 220 & 20617 & 137 & 10.8 & 0.04 \\
\hline 240 & 20641 & 273 & & \\
\hline
\end{tabular}


type 4 (102 km/h trains). As shown in Table 3 and Fig. 6, with increasing the percentage of lower speed trains on the route, the capacity decreases to 110 trains per day. Fig. 6 shows the line section capacity of Tehran-Zanjan route in some different proportional mix of train types. For example, if the proportional mix of train types 3 and 4 are equal to 50 percent, the line section capacity is equal to 114 trains per day.

As the combination of different train types increases, more interference is generated. In this case, trains require overtaking and crossing, and the mixture reduces the railway capacity. The presented model considers

Table 3. The critical line section capacity

\begin{tabular}{cccc}
\hline Scenario & $\begin{array}{c}\text { Train type 3 } \\
(100 \mathrm{~km} / \mathrm{h})\end{array}$ & $\begin{array}{c}\text { Train type } 4 \\
(102 \mathrm{~km} / \mathrm{h})\end{array}$ & $\begin{array}{c}\text { Line capacity } \\
\text { (trains per day) }\end{array}$ \\
\hline 1 & 13 & 104 & 117 \\
\hline 2 & 24 & 92 & 116 \\
\hline 3 & 47 & 68 & 115 \\
\hline 4 & 59 & 55 & 114 \\
\hline 5 & 71 & 42 & 113 \\
\hline 6 & 83 & 29 & 112 \\
\hline 7 & 95 & 14 & 111 \\
\hline 8 & 99 & 11 & 110 \\
\hline
\end{tabular}

overtaking issues, crossing issues, the proportional mix of trains and siding tracks on each line section. Capacity tends to increase non-linearly with small incremental changes in the parameters. In each scenario, the model maximizes the number of trains in arriving at their destinations taking into account each priority. Therefore, this aim causes of increasing of total train waiting time. That is because of trains with lower speed wait in the siding track for transferring higher speed train types.

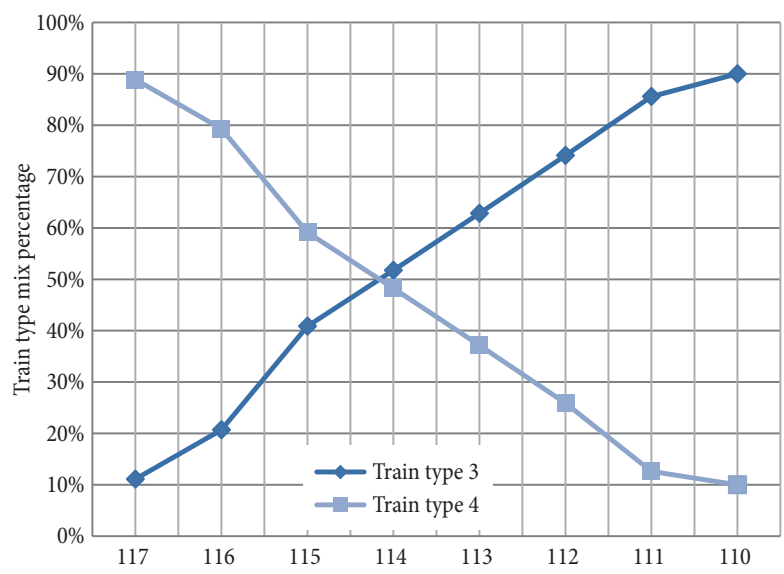

Fig. 6. The impact of different train types on railway line capacity

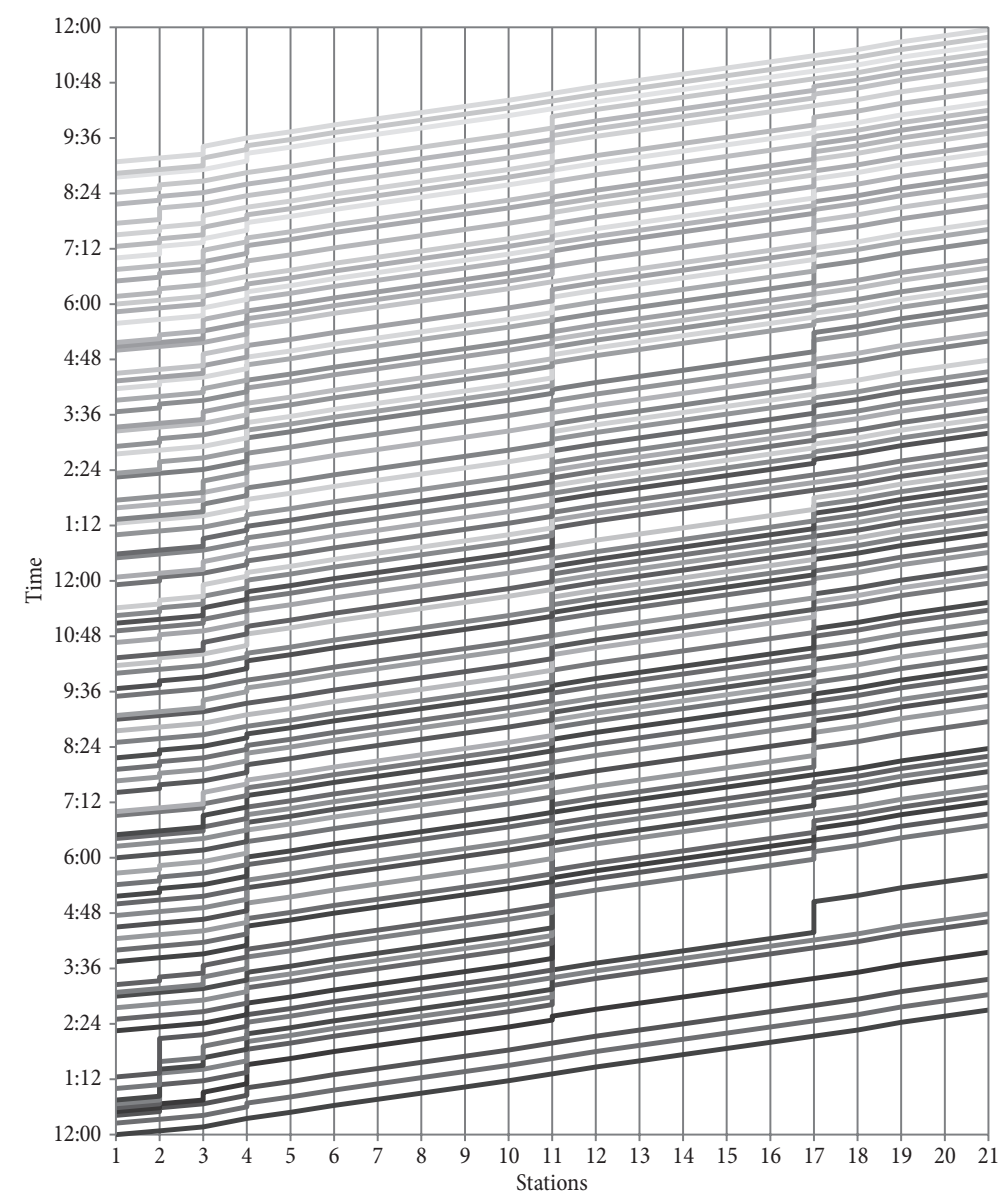

Fig. 5. The train timetable diagram 


\section{Conclusions}

In this paper, the optimization models for calculation of railway line and line section capacity and evaluation of train type interactions on railway line capacity using a discrete-time network are presented.

The proposed models consider infrastructure, traffic, and operating parameters.

For representation of railway line, a space-time network is proposed. This space-time network representation models railway line and reduces the number of decision variables. Since each train in each time unit travels on the line section or waits at the nodes, two sets of arcs are defined.

The objective of the proposed model is to maximize the number of trains in railway line and line section in the specific period. The inputs of the models are railway line and train type attributes, which are typically available to planners.

These models consider train overtaking and crossing issues, train type priorities, infrastructure, and operating parameters in aggregate. The proposed model did not need a detailed timetable for capacity evaluation. In addition, the important output of the proposed model is saturated timetable. In order to evaluate the proposed model, it is implemented in Iran Railways.

The results show that the capacity is increased, but waiting time increases and then decreases with raising the average velocity of trains. In addition, with increasing the percentage of lower speed trains on the route, railway line capacity decreases. The capacity tends to increase non-linearly with small incremental changes in the parameters. The results show the applicability of the proposed model in planning stage.

\section{References}

Abril, M.; Barber, F.; Ingolotti, L.; Salido, M. A.; Tormos, P.; Lova, A. 2008. An assessment of railway capacity, Transportation Research Part E: Logistics and Transportation Review 44(5): 774-806. http://dx.doi.org/10.1016/j.tre.2007.04.001

Burdett, R. L.; Kozan, E. 2006. Techniques for absolute capacity determination in railways, Transportation Research Part B: Methodological 40(8): 616-632. http://dx.doi.org/10.1016/j.trb.2005.09.004

Dingler, M. H.; Lai, Y. C.; Barkan, C. P. L. 2009. Impact of train type heterogeneity on single-track railway capacity, Transportation Research Record 2117: 41-49. http://dx.doi.org/10.3141/2117-06

Hansen, I. A. 2010. Railway network timetabling and dynamic traffic management, International Journal of Civil Engineering 8(1): 19-32.

Harrod, S. 2009. Capacity factors of a mixed speed railway network, Transportation Research Part E: Logistics and Transportation Review 45(5): 830-841. http://dx.doi.org/10.1016/j.tre.2009.03.004

Khadem-Sameni, M.; Preston, J. M.; Armstrong, J. 2010. Railway capacity challenge: measuring and managing in Britain, in Proceedings of the American Society of Mechanical Engineers Joint Rail Conference, 27-29 April 2010, Urbana, Vol. 2, 571-578.
Kontaxi, E.; Ricci, S. 2011. Calculation of railway network capacity: comparing methodologies for lines and nodes, in RAILROME 2011: 4th International Seminar on Railway Operations Modelling and Analysis (Book of Abstracts), 16-18 February 2011, Rome, Italy, 31-31.

Lai, Y.-C.; Barkan, C. P. L. 2011. Comprehensive decision support framework for strategic railway capacity planning, Journal of Transportation Engineering 137(10): 738-749. http://dx.doi.org/10.1061/(ASCE)TE.1943-5436.0000248

Landex, A. 2009. Evaluation of railway networks with single track operation using the UIC 406 capacity method, Networks and Spatial Economics 9(1): 7-23. http://dx.doi.org/10.1007/s11067-008-9090-7

Landex, A.; Kaas, A. H.; Schittenhelm, B.; Schneider-Tilli, J. 2006. Evaluation of railway capacity, in Artikelsamling fra Trafikdage på Aalborg Universitet 2006. 28-29 August 2006, Aalborg, Denmark, 232-253.

Libardo, A.; Pellegrini, P.; Salerno, G. 2011. Capacity in railway junctions and optimal route management, in RAILROME 2011: 4th International Seminar on Railway Operations Modelling and Analysis (Book of Abstracts), 16-18 February 2011, Rome, Italy, 54-54.

Merel, A.; Gandibleux, X.; Demassey, S.; Lusby, R. 2009. An improved upper bound for the railway infrastructure capacity problem on the Pierrefitte-Gonesse junction, in 10ème Congrès de la Société Française de Recherche Opérationnelle et d'Aide à la Décision (ROADEF'09), 10-12 February 2009, Nancy, France, 62-76.

Pachl, J.; White, T. 2004. Analytical capacity management with blocking times, in 83rd Annual Meeting of the Transportation Research Board: Compendium of Papers, 11-15 January 2004, Washington, D.C. 14 p. (CD).

UIC. 2004. UIC Code 406: Capacity. International Union of Railways. 22 p. 